\title{
Birthweights in Same-sex and Opposite-sex Twin Pregnancies
}

\author{
Susan A. Treloar and John B. Whitfield \\ Queensland Institute of Medical Research
}

The suggestion made by James (2002), that male birthweights in opposite-sex dizygotic twin pairs are greater, and female birthweights are less, than would be predicted from birthweights in same-sex dizygotic twin pairs, was tested against data collected from Australian twins.

Self-reported birthweights were taken from postal questionnaire data on male-male (MM), female-female (FF) and male-female (MF) dizygotic twin pairs (Treloar et al., 2000; Whitfield et al., 2001). Zygosity was determined from the subjects' responses to questions about physical similarity and the inability of others to tell them apart, supplemented by blood group typing. The mean reported weights by sex and zygosity were calculated, and within-pair differences were calculated for MF pairs. The mean within-pair (MF) difference was compared with the difference in mean birthweights for males and females from MM and FF pairs.

Birthweights by sex and zygosity are shown in Table 1. As predicted, male-female twin pregnancies gave rise to males who were heavier than males from same-sex twin pregnancies. However, a similar trend applied to the females, although the group means were not significantly different in either case (unpaired $t$ test, with unequal variances between the groups). Within-pair comparisons of the MF pairs showed that the average male-female difference was $147 \pm 14$ (SEM) grams, which is practically identical to the 145 gram difference in the male and female means from same-sex pairs. These conclusions are subject to any possible bias in self-reports from members of opposite-sex pairs.

Therefore James' suggestion is not supported by our data, as the males from opposite-sex pairs had increased birthweights (as predicted) but their female co-twins were also slightly heavier (by 14 grams) than females from same-sex pairs. Within oppositesex twin pregnancies, males gain approximately 17 grams in weight; but not at the expense of their sisters. Our data unfortunately do not include length of gestation of these twin pairs, but our findings are consistent with the possible explanation (Loos et al., 2001) that opposite-sex twin pregnancies have a longer mean gestation compared to the gestation period of same-sex twins.

\section{References}

James,W. H. (2002). Birthweight in dizygotic twins (letter). Twin Research, 5, 309.

Loos, R. J. F., Derom, C., Eeckels, R., Derom, R., \& Vlietinck, R. (200I). Length of gestation and birthweight in dizygotic twins. Lancet, 358, 560-56I.

Treloar, S. A., Sadrzadeh, S., Do, K. A., Martin, N. G., \& Lambalk, C. B. (2000). Birth weight and age at menopause in Australian female twin pairs: Exploration of the fetal origin hypothesis. Human Reproduction, 15, 55-59.

Whitfield, J. B., Treloar, S. A., Zhu, G., \& Martin, N. G. (200I). Genetic and non-genetic factors affecting birthweight and adult body mass index. Twin Research, 4, 365-370.

\section{Table 1}

Mean $( \pm S D)$ Self-reported Birthweights by Sex and Zygosity.

\begin{tabular}{lcc}
\hline & Male birthweight (grams) & Female birthweight (grams) \\
\hline MM DZ pairs $(N=290)$ & $2675 \pm 602$ & N/A \\
MF DZ pairs $(N=908)$ & $2692 \pm 611$ & $2544 \pm 609$ \\
FF DZ pairs $(N=808)$ & N/A & $2530 \pm 586$ \\
Difference in means & & \\
(opposite-sex) - (same-sex) & +17 & +14 \\
$p$ (one-tail) & 0.282 & 0.766
\end{tabular}

